\title{
Polyhydroxy alkanoates Production by Newly Isolated Bacteria Serratia ureilytica Using Volatile Fatty Acids as Substrate: Bio-Electro Kinetic Analysis
}

\author{
M Venkateswar Reddy and S Venkata Mohan* \\ Bioengineering and Environmental Centre (BEEC), CSIR-Indian Institute of Chemical Technology (CSIR-IICT), Hyderabad-500 007, India
}

\begin{abstract}
Polyhydroxy alkanoates (PHAs) show physical and material properties similar to conventional plastics, so these are considered as an alternative to non-biodegradable plastics. In the present study, PHA production using Serratia ureilytica, a newly isolated bacterial strain from PHA producing bioreactor was investigated using volatile fatty acids as substrate at four different organic loading rates (OLRs, OLR1-OLR4). Among four OLRs, S. ureilytica showed highest PHA production (51\% dry cell weight) with OLR2 at $24 \mathrm{~h}$, but it showed highest substrate removal with OLR1 (84\%). PHA composition showed the presence of co-polymer, poly (3hydroxybutyrate-co-3hydroxyvalerate), $\mathrm{P}$ (3HBco-3HV). In cyclic voltammetry analysis the activity of specific redox mediators was observed at $24 \mathrm{~h}$ and $36 \mathrm{~h}$ of PHA production process. Lower Tafel slopes in bio-electrokinetic analysis also supported the decrease in electron losses during PHA synthesis. Bioprocess evaluation and enzymatic activities showed good correlation with PHA production.
\end{abstract}

Keywords: Bioplastics; Dehydrogenase; Chromatography; Cyclic voltammetry $(\mathrm{CV}) ; \mathrm{P}(3 \mathrm{HB}-c o-3 \mathrm{HV})$

\section{Introduction}

Accumulation of synthetic plastics in the environment is a worldwide problem. The crude oil equal to 18 million barrels were used to produce 2 million polyethylene terephthalate bottles in 2005 . Along with the environmental problems, rapid diminution of natural resources, is forcing research to focus on alternatives to petroleum based synthetic plastics [1]. Bioplastics in the form of polyhydroxy alkanoates (PHA) are an alternative to synthetic plastics and use of these bioplastics could lower the contribution of synthetic plastics to municipal landfills by about $20 \%$ of the total waste by volume and $10 \%$ by weight [2]. PHA are the high molecular weight $(50-100 \mathrm{KDa})$ carbonaceous, cellular reserve products synthesized by microbes as storage granules $[3,4]$ during excess supply of carbon and the lack of one or more essential nutrients, e.g., oxygen, nitrogen, phosphorus, sulfur, and trace elements such as magnesium, calcium, and iron [5]. PHA has attracted commercial interest as plastic materials, because of their similarities in physical properties with synthetic plastics. Usage of PHA in the field of packaging, medical, pharmaceutical, agricultural and fisheries has recently gained enormous attention due to their biodegradability and biocompatibility [6]. PHA can also be used for manufacturing disposable articles such as shampoo bottles and cosmetic materials. In the medical field, PHA is widely used in orthopedic applications including sutures, meniscus regeneration devices, fixation rods, bone plates and bone fracture fixation. Hence, PHA and modified PHA are being considered as solutions for the ever increasing biomedical challenges [6].

PHA are synthesized and stored intracellularly by a wide variety of bacteria such as, Bacillus sp., Pseudomonas sp., Azobacterium and many recombinant strains through the fermentation of sugars, lipids, alkanes, alkenes and alkanoic acids [7-11]. Among the pure cultures, the bacteria Cupriavidus necator has been widely studied because of its potential in producing significant amounts of PHA from simple organic carbon substrates such as glucose, acetic acid [12], waste glycerol [13], and in-organic carbon source like carbon dioxide [14]. Recombinant E. coli harboring the Cupriavidus necator PHA biosynthetic genes were also able to accumulate PHB with a yield of $80-90 \%$ DCW [15]. The physical and chemical properties of PHAs are affected by the monomer composition. The monomer composition of the polymer depends on type of bacteria, media ingredients, fermentation conditions, mode of fermentation, and recovery methods [8].

Many studies were reported on the utilization of individual volatile fatty acids [16,17] and mixed volatile fatty acids [18-20] for the production of PHA using various bacteria. However, there are no literature reports on production of PHA using volatile fatty acids with Serratia urelytica, even though this group of organisms have commercial importance and used in the production of commercially valuable products like enzymes, biosurfactants, bioplastics, and antioxidants. Hence, in this communication, mixture of three volatile fatty acids (acetate, propionate and butyrate) were used as substrate to know the potentially of new bacterial isolate $S$. ureilytica as biocatalyst for PHA production. Cyclic voltammetry and bio-electrokinetic analysis was also done to know the activity of specific redox mediators involved, and electron losses occurring during the PHA production process.

\section{Materials and Methods}

\section{Isolation of S. ureilytica}

The bacteria S. ureilytica was isolated by direct plating and enrichment techniques from a PHA producing bioreactor which was operated by using mixed bacterial culture as biocatalyst and different

*Corresponding author: S Venkata Mohan, Bioengineering and Environmental Centre (BEEC), CSIR-Indian Institute of Chemical Technology (CSIR-IICT), Hyderabad-500 007, India, Tel: 0091-40-27191664; Fax: 0091-40-27191664 E-mail:vmohan_s@yahoo.com

Received December 25, 2014; Accepted January 30, 2015; Published February 06, 2015

Citation: Venkateswar Reddy M, Venkata Mohan S (2015) Polyhydroxy alkanoates Production by Newly Isolated Bacteria Serratia ureilytica Using Volatile Fatty Acids as Substrate: Bio-Electro Kinetic Analysis. J Microb Biochem Technol 7: 026-032. doi:10.4172/1948-5948.1000177

Copyright: @ 2015 Venkateswar Reddy M, et al. This is an open-access article distributed under the terms of the Creative Commons Attribution License, which permits unrestricted use, distribution, and reproduction in any medium, provided the original author and source are credited 
Citation: Venkateswar Reddy M, Venkata Mohan S (2015) Polyhydroxy alkanoates Production by Newly Isolated Bacteria Serratia ureilytica Using Volatile Fatty Acids as Substrate: Bio-Electro Kinetic Analysis. J Microb Biochem Technol 7: 026-032. doi:10.4172/1948-5948.1000177

wastewaters as substrates. The isolated S. ureilytica as used as biocatalyst for PHA production.

\section{Substrate}

Designed synthetic wastewater (DSW) with varying organic loading rates (OLR1, 0.66; OLR2, 1.32; OLR3, 1.98; OLR4, $2.64 \mathrm{~kg} \mathrm{COD} / \mathrm{m}^{3}$ day) was used as substrate. One liter of designed synthetic wastewater was prepared by adding different concentrations of volatile fatty acids, nutrients and minerals in sterile water [20]. In all the OLRs, acetate was used as major carbon source along with aliquots of propionic acid and butyric acid. The carbon source composition of DSW in OLR1 (acetate, $2.5 \mathrm{~g} / \mathrm{l}$; propionate, $0.25 \mathrm{~g} / \mathrm{l}$; butyrate, $0.25 \mathrm{~g} / \mathrm{l}$, COD, $3.15 \pm 0.1$ $\mathrm{g} / \mathrm{l}$ ), OLR2 (acetate, $5 \mathrm{~g} / \mathrm{l}$; propionate, $0.5 \mathrm{~g} / \mathrm{l}$; butyrate, $0.5 \mathrm{~g} / \mathrm{l}$, COD, $6.05 \pm 0.2 \mathrm{~g} / \mathrm{l}$ ), OLR3 (acetate, $7.5 \mathrm{~g} / \mathrm{l}$; propionate, $0.75 \mathrm{~g} / \mathrm{l}$, butyrate, $0.75 \mathrm{~g} / \mathrm{l}, \mathrm{COD}, 9.12 \pm 0.2 \mathrm{~g} / \mathrm{l}$ ) and OLR4 (acetate, $10 \mathrm{~g} / \mathrm{l}$, propionate, $1 \mathrm{~g} / \mathrm{l}$, butyrate, $1 \mathrm{~g} / \mathrm{l}$, COD, $12.24 \pm 0.3 \mathrm{~g} / \mathrm{l}$ ) was varied accordingly. Substrate was autoclaved before feeding to the reactors.

\section{Experimental design}

The feasibility of PHA production was evaluated using S. ureilytica as a biocatalyst with DSW as substrate. Batch experiments were performed in separate reactors operated in suspended growth mode. The total/working volume of $250 / 100 \mathrm{ml}$ was used and operated with continuous mixing at $120 \mathrm{rpm}$ at ambient temperature $\left(30^{\circ} \mathrm{C}\right)$. The reactors containing $100 \mathrm{ml}$ of substrate was inoculated with $2 \%$ of overnight activated $S$. ureilytica prior to startup. The initial $\mathrm{pH}$ of the substrate was adjusted to 7 using $1 \mathrm{~N} \mathrm{NaOH}$. Samples were collected at different time intervals for extraction of PHA, and for analysis of substrate degradation, dehydrogenase enzyme activity, and bio-electro kinetic analysis. Quantitative analysis was carried out in triplicate and the average values were presented.

\section{Analysis}

Bioprocess was monitored by evaluating process performance based on $\mathrm{pH}$, volatile fatty acids (VFA), chemical oxygen demand (COD, closed refluxing method), and substrate degradation rate (SDR) estimation according to the standard methods [21]. Dehydrogenase enzyme activity of biocatalyst was estimated by using the method described previously [22].

PHA: Extraction of PHA was performed according to the procedure reported previously $[23,24]$. The biomass pellet was separated from the substrate by centrifugation $\left(6000 \times \mathrm{g}\right.$ for $6 \mathrm{~min}$ at $\left.10^{\circ} \mathrm{C}\right)$ and the resulting pellet was washed with acetone and ethanol separately to remove unwanted materials. The pellet was suspended in $4 \%$ sodium hypochlorite and incubated at room temperature for $3 \mathrm{~h}$. The resulting mixture was centrifuged and the supernatant was discarded. The pellet with lysed cells after washing simultaneously with acetone and ethanol was dissolved in hot chloroform and was passed through glass fiber filter $(0.45 \mu \mathrm{m}$ pore size $)$ to separate the polymer from cell debris. Chloroform filtrate obtained was purified using cold ethanol and was used to estimate PHA concentration. Presence of PHA in the extracted sample was confirmed by FT-IR spectroscopic analysis (Thermo Nicolet Nexus 670; KRS-5; spectral resolution, $4 \mathrm{~cm}^{-1}$; range, 4000-400 $\mathrm{cm}^{-1}$ ). PHA quantification was carried out by high performance liquid chromatography (HPLC; Shimadzu LC10A) according to procedure outlined by Karr et al., (1988) [25]. Standard curve was plotted using pure $\mathrm{P}$ (3HB-co-3HV co-polymer; natural origin, Aldrich).

Thermogravimetric analysis (TGA): PHA samples were subjected to thermogravimetric analysis (TGA) (Mettler Toledo TGA/SDTA851
Switzerland) to determine the decomposition temperature of PHA. All analyses were performed with $5 \mathrm{mg}$ of sample in an aluminium pan over a temperature range of $50-900^{\circ} \mathrm{C}$ under nitrogen atmosphere, at a heating rate of $5^{\circ} \mathrm{C} \mathrm{min}^{-1}$.

Bacterial growth and dry cell weight (DCW) determination: A loop full of isolated strain was inoculated into $20 \mathrm{ml}$ of nutrient broth in a $100 \mathrm{ml}$ flask, and the flask was kept at $30^{\circ} \mathrm{C}$ for overnight. $2 \mathrm{ml}$ of the overnight grown culture was then inoculated to $100 \mathrm{ml}$ of synthetic acids in $250 \mathrm{ml}$ flasks. Growth was monitored spectrophotometrically (GE, GeneQuant-1300) by measuring the absorbance at $600 \mathrm{~nm}$, and dry cell weight was measured periodically at different OLRs by using gravimetric method. The cells were harvested by centrifugation, washed twice with phosphate buffer and dried at $80^{\circ} \mathrm{C}$ until a stable weight was reached.

\section{Cyclic voltammetry}

Electron discharge profiles of the biocatalyst during PHA production was evaluated by employing cyclic voltammetry (CV) using electrochemical cell having platinum and graphite rod as working and counter electrodes, respectively against $\mathrm{Ag}-\mathrm{AgCl}(\mathrm{S})$ reference electrode [26]. Voltammograms were recorded by potentio stat-glavanostat system (Autolab, PGSTAT12, Ecochemie) by applying a potential ramp at a scan rate of $30 \mathrm{mV} / \mathrm{s}$ over the range of +0.5 to $-0.5 \mathrm{~V}$ to the working electrode. All the electrochemical assays were performed with S. ureilytica in whole cell form using designed synthetic wastewater as electrolyte. During potential sweep, the potentiostat measures the current resulting from the applied potential and the values were used to plot the voltammogram taking current vs. the applied potential. Change in current (i) with respect to the operational conditions and time could be visualized in CV. Tafel analysis was made from the voltammetric profiles obtained at maximum performance using GPES (version 4.0) software and conclusions were drawn in terms of Tafel slope and polarization resistance.

\section{Results and Discussion}

\section{Identification of Serratia ureilytica}

The 16S rRNA nucleotide sequence identified in this study was deposited in the GenBank database under accession number HE612873. The sequence was aligned with the closest matches found in the GenBank database with the CLUSTALW function of Molecular Evolutionary Genetics Analysis package (MEGA). Neighbour-joining phylogenetic tree was constructed with the MEGA version 4.0. The isolated strain phylogenetically relates to genus Enterobacteriaceae of the subclass of Proteobacteriaand formed a monophyletic lineage (Figure 1). Sequence similarity calculations after neighbor-joining analysis indicated that the closest relatives of HE612873 strain are $S$. ureilytica (100\%), Enterobacter sp. (99\%), Enterobacter sp. F5jun (99\%), Klebsiella sp. FF2(99\%) and Bacterium YX117S (99\%). Growth study results of $S$. ureilytica depicted a long lag phase up to $5 \mathrm{~h}$, whereas

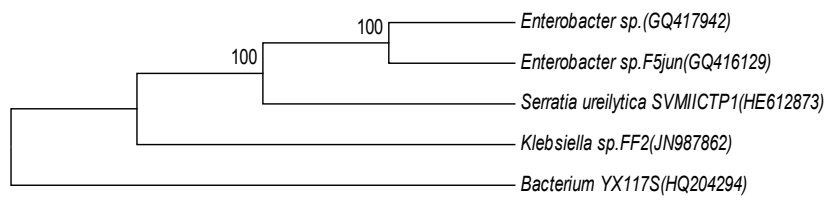

Figure 1: Neighbor-joining tree constructed using Mega 4.0 showing phylogenetic relationships of 16S rRNA sequences from closely related sequences from Gen Bank. 
the exponential phase was up to $22 \mathrm{~h}$, followed by stationary phase. The three phases of the curve are clearly distinguished in the bacterial growth. The slope of the exponential growth phase resulted in specific growth rate at that substrate concentration. Maximum specific growth rate $\left(\mu_{\max }\right)$ from the Monods' curve was estimated using two linearized methods viz., Lineweaver-Burk, and Eadie-Hofstee. From the kinetic data, it is observed that the according to Lineweaver-Burk method the $\mu_{\max }$ value was $0.36 \mathrm{~h}^{-1}$, and according to Eadie-Hofstee method the $\mu_{\max }$ value was $0.34 \mathrm{~h}^{-1}$.

\section{PHA production from volatile fatty acids}

This study aimed to evaluate the PHA production from a volatile fatty acids mixture using newly isolated bacteria $S$. ureilytica. Volatile fatty acids have already been applied as organic carbon source for PHA production using several bacteria. Wang and $\mathrm{Wu},(2000)$ reported that the bacteria Alcaligenes eutrophus produced 55\% of PHB using sodium acetate as sole carbon source at nutrient rich conditions [16]. Cyanobacterium Nostoc muscorum, produced $43 \%$ of PHB using $4 \mathrm{~g} / \mathrm{L}$ acetate as carbon source [17]. Du et al., (2001) conducted experiments with Ralstonia eutropha for the production of PHA using three types of volatile fatty acids i.e., acetic acid, propionic acid, and butyric acid, and they reported that Ralstonia eutropha produced highest PHA when butyric acid was used as carbon source [27]. There are many reports on the utilization of volatile fatty acids by various bacteria for the production of PHA, but there are no studies on the production of PHA using volatile fatty acids with S.urelytica, even though this group of organisms has commercial importance and used in the production of commercially valuable products like enzymes, biosurfactants, bioplastics, and anti-oxidants. Lugg et al., (2008) reported that a strain of Serratia sp. showed $55 \%$ of PHB per gram of biomass dry weight when incubated in the presence of citrate and glycerol 2-phosphate as substrates [28]. Nalini and Parthasarathi (2014) reported the production of biosurfactants by SerratiarubidaeaSNAU02 under solid state fermentation using mahua oil cake as a substrate [29]. Wang et al., (2009) used the bacteria Serratia ureilytica for the production of antioxidants with anti-tumor activity and enzymes like protease and chitinase by using squid pen as substrate [30].So in the present study we used the bacteria Serratia ureilytica for the production of PHA using volatile fatty acids as substrate. This organism is facultatively aerobic, Gram-negative, motile, non-fluorescent, non-pigmented, straight rods. Grows between $8^{\circ} \mathrm{C}$ to $43^{\circ} \mathrm{C}$ over a $\mathrm{pH}$ range of $5-11$ in nutrient broth media, and can tolerate up to $1.4 \mathrm{M} \mathrm{NaCl}$. These bacteria can utilize urea as a sole nitrogen source for its growth and has the ability to hydrolyze urea to ammonia [31]. Ye et al. (2012) reported $37 \%$ production of PHA using mixed volatile fatty acids recombinant Escherichia coli [32].

In the present study polyhydroxy alkanoates were accumulated by $S$. ureilytica when mixture of volatile fatty acids at four OLRs was supplied to the culture. PHA was accumulated to different levels ranging from $32 \%$ to $51 \%$ DCW (Figure $2 a$ ). The best accumulation of biopolymer from substrate was achieved at OLR2 (51\%), and it was 2.8 folds higher in comparison with PHA accumulation at OLR1 (32\%), which also supported by the lowest levels of growth at OLR1. The best biomass yields were achieved at OLR2 (3.9 g/l), and it was 1.2 fold to 1.8 fold higher in comparison with all other OLRs. The PHA accumulation capacity of S. ureilytica from the OLR3 and OLR4 was 1.2 fold and 1.5 fold lower than the OLR2 respectively (Figure 2a). Variation in PHA production visualized during the study might be due to the substrate load which influenced the process performance. The time taken for maximum PHA production was found to increase with increasing
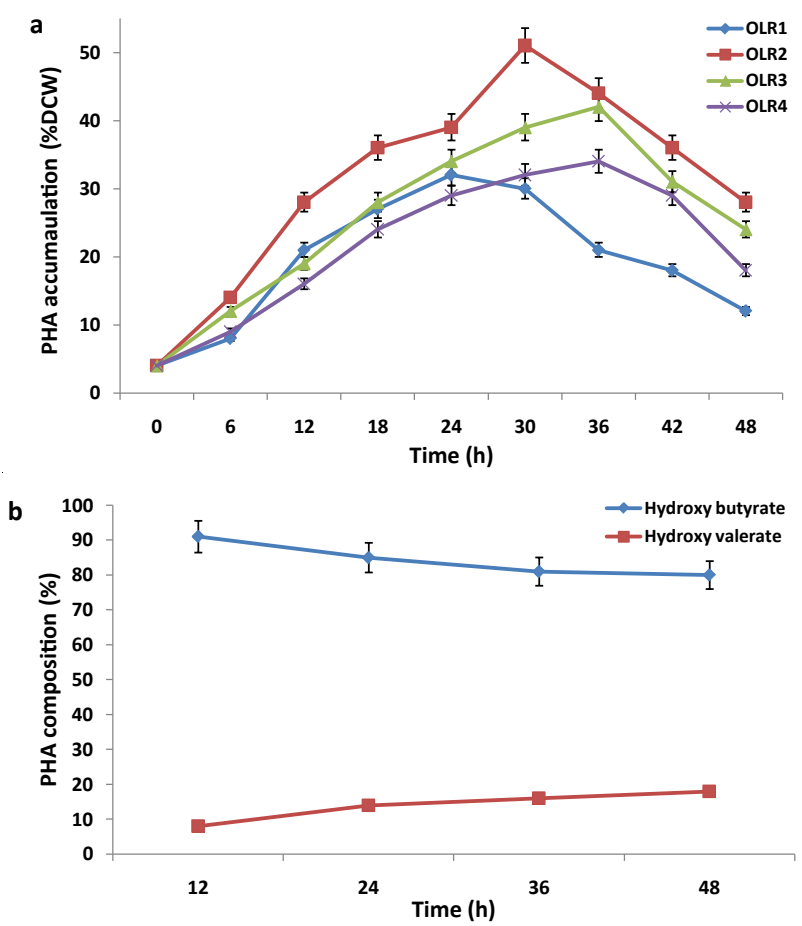

Figure 2: (a) PHA produced from Serratiaureilytica using volatile fatty acids at different OLRs as substrate; (b) Composition of PHA recovered from Serratiaureilytica using volatile fatty acids at OLR2.

organic load i.e., OLR1 at $18 \mathrm{~h}$, OLR2 at $24 \mathrm{~h}$, OLR3 and OLR4 at 36 h. Higher OLR took more time for maximum PHA production due to the availability of more substrate, while, lower OLR showed maximum PHA accumulation in shorter time due to the lower substrate availability. After reaching maximum concentration, production capacity of biocatalyst showed a gradual decrement up to end of the experiment $(48 \mathrm{~h})$ for all the OLR studied. OLR1 showed low PHA production than other OLRs due to insufficient supply of substrate. Conversely in the case of OLR4 high substrate concentration caused load shock and suppressed the bacterial growth thereby lowering PHA production. The biocatalyst showed highest PHA production at OLR2 signifying that this concentration was ideal for bacterial growth and PHA production which was further supported by high dehydrogenase enzyme activity.

Composition of biopolymer:Composition of PHA was analyzed using HPLC. HPLC analysis of PHA sample in $0.014 \mathrm{~N} \mathrm{H}_{2} \mathrm{SO}_{4}$ showed two peaks at retention times of $33 \mathrm{~min}$ and $40 \mathrm{~min}$ matching with the standard co-polymer $\mathrm{P}(3 \mathrm{HB}-\mathrm{co}-3 \mathrm{HV})$. Composition of PHA based on co-polymer, $\mathrm{P}(3 \mathrm{HB}-\mathrm{co}-3 \mathrm{HV})$ synthesis was determined for OLR2 at different time intervals ( $12 \mathrm{~h}, 24 \mathrm{~h}, 36 \mathrm{~h}, 48 \mathrm{~h}$ ) (Figure $2 \mathrm{~b}$ ). The polymer obtained, showed high HB content and low HV content for all the time intervals. The HV content showed an increment with increase in time. This is due to the utilization of propionate for HV formation, which can be further supported by determining the volatile fatty acids composition using HPLC analysis. At $12 \mathrm{~h}$ polymer showed higher HB content (91\%) than $\mathrm{HV}(8 \%)$, followed by $24 \mathrm{~h}(\mathrm{HB}, 85 \%$; HV $14 \%)$, $36 \mathrm{~h}(\mathrm{HB}, 81 \%$; HV 16\%) and $48 \mathrm{~h}$ (HB, 80\%; HV 18\%). Biocatalyst showed high $\mathrm{HB}$ content at all the time intervals due to the presence of high amounts of acetic acid and low amount of butyric and propionic acids in VFA composition. Homo-polymer, PHB is highly crystalline, 
Citation: Venkateswar Reddy M, Venkata Mohan S (2015) Polyhydroxy alkanoates Production by Newly Isolated Bacteria Serratia ureilytica Using Volatile Fatty Acids as Substrate: Bio-Electro Kinetic Analysis. J Microb Biochem Technol 7: 026-032. doi:10.4172/1948-5948.1000177

stiff and brittle with low impact strength. Co-polymer, $\mathrm{P}(3 \mathrm{HB}-c o-3 \mathrm{HV})$ has better physical and thermal properties, which depend on the HV unit. If HV fraction increases, melting temperature decreases without affecting the degradation temperature, thus providing a polymer with structural properties and process ability similar to conventional plastics [33]. Therefore the presence of high HB content and low HV content supports that the PHA obtained is biodegradable and can be used as an alternative to synthetic plastics.

Biopolymer characterization: The biopolymer produced by $S$. ureilytica was characterized by different techniques. FTIR analysis revealed the presence of different conformational bands related to PHA (data not shown). The presence of absorption band at $1019 \mathrm{~cm}^{-1}$ represented $\mathrm{C}-\mathrm{O}-\mathrm{C}$ chemical groups of the polymer. The signals in the regions of $3422 \mathrm{~cm}^{-1}, 2925 \mathrm{~cm}^{-1}$ and $1448 \mathrm{~cm}^{-1}$ corresponds to the stretching of $\mathrm{CH}_{3}$ asymmetric, $\mathrm{CH}_{2}$ anti-symmetrical and methyne $(-\mathrm{CH})$ group respectively. The thermal stability of the polymers was analyzed by thermogravimetric analysis. Sample curve of PHA obtained from S. ureilytica was given in Figure 3. The temperature at $5 \%$ weight loss was used as the decomposition temperature $(T d)$ to evaluate the thermal stability. While the decomposition of PHA starts at $200^{\circ} \mathrm{C}$, and derivative thermo-gravimetric (DTG) analysis showed that the temperature corresponding to maximum degradation rate, i.e. Dmax was $270^{\circ} \mathrm{C}$ for PHA.

\section{Bioprocess monitoring}

Volatile fatty acids concentration: The substrate $\mathrm{pH}$ was adjusted to 7 before feeding to the reactors. The substrate $\mathrm{pH}$ showed an increasing trend with time which might be due to the utilization of volatile fatty acids by $S$. ureilytica. At $48 \mathrm{~h}$, OLR1 showed highest increment in $\mathrm{pH}$ (from 7 to 8.6), followed by OLR2 (7 to 8.3), OLR3 (7 to 8.1) and OLR4 (7 to 7.6) (Figure 4a). Changes in the $\mathrm{pH}$ values were correlated well with the volatile fatty acids removal pattern. Volatile fatty acids are the major precursor components involved in PHA production. Initial volatile fatty acids concentrations were varied for all the OLRs i.e., OLR1-3022 mg/l; OLR2-6056 mg/l; OLR3-9128 $\mathrm{mg} / \mathrm{l}$, and OLR4-12248 mg/l (Figure 4b). In all the OLRs, volatile fatty acids showed gradual decrement up to end of the experiment, which might be due to their continuous utilization by $S$. ureilytica for the

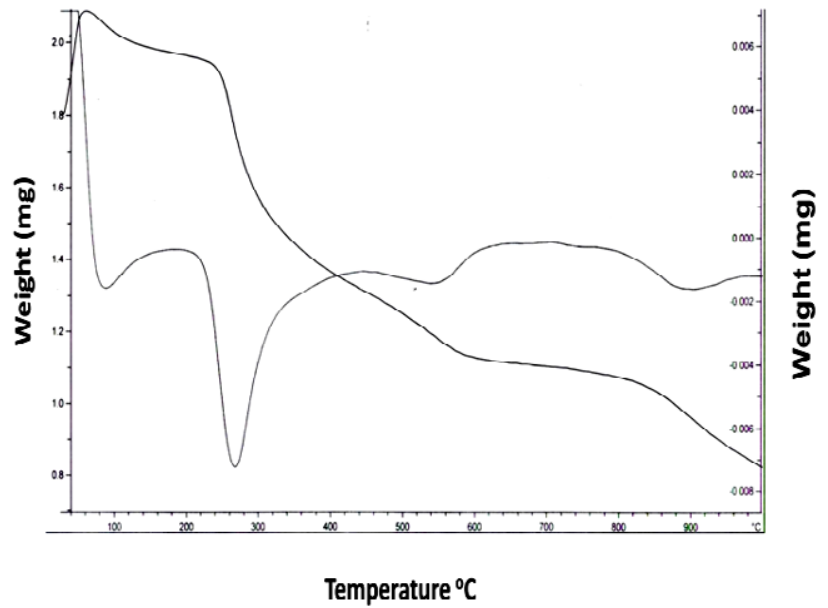

Figure 3: TGA thermogram of PHA produced from Serratiaureilytica using volatile fatty acids as substrate.
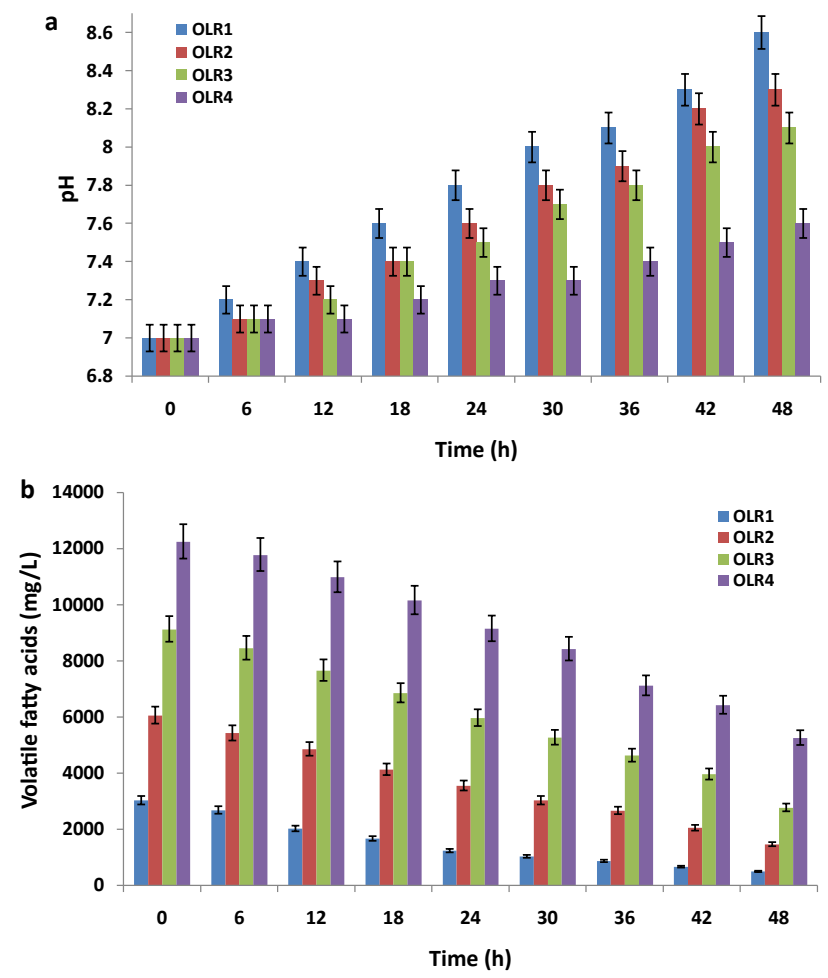

Figure 4: Bioprocess monitoring analysis of (a) pH and (b) VFAestimated by standard method using volatile fatty acids as substrate at different OLRs.

\begin{tabular}{|c|c|c|c|}
\hline \multirow{2}{*}{ Time (h) } & \multicolumn{3}{|c|}{ Volatile fatty acids (mg/l) } \\
\hline & Acetic acid & Butyric acid & Propionic acid \\
\hline 0 & $4800 \pm 320$ & $490 \pm 45$ & $480 \pm 52$ \\
\hline 12 & $4254 \pm 246$ & $284 \pm 27$ & $265 \pm 24$ \\
\hline 24 & $3212 \pm 165$ & $178 \pm 28$ & $134 \pm 18$ \\
\hline 36 & $2234 \pm 124$ & $102 \pm 26$ & $94 \pm 14$ \\
\hline 48 & $1456 \pm 114$ & $51 \pm 14$ & $32 \pm 12$ \\
\hline
\end{tabular}

Table 1: Composition of volatile fatty acids estimated by HPLC during different time intervals at OLR2.

production of PHA. Among all the OLRs, S. ureilytica at OLR1 showed highest volatile fatty acids removal efficiency (84\%), followed by OLR2 (75\%), OLR3 (69\%), and OLR4 (57\%). Individual volatile fatty acids composition of the substrate at OLR2 was analyzed at specific time intervals ( $0 \mathrm{~h}, 12 \mathrm{~h}, 24 \mathrm{~h}, 36 \mathrm{~h}, 48 \mathrm{~h})$ using HPLC (Table 1). HPLC result showed that among three acids, acetic acid showed maximum removal (3344 mg/l), followed by propionic acid (448 $\mathrm{mg} / \mathrm{l})$, and butyric acid $(439 \mathrm{mg} / \mathrm{l})$ at $48 \mathrm{~h}$ of the experimental period.

Substrate removal: Substrate removal efficiency was evaluated in terms of COD removal. Substrate degradation correlated well with PHA production. Increment in substrate removal was noticed with operation time irrespective of the OLR studied, suggesting the efficiency of the system towards treatment. S. ureilyticashowed highest COD removal efficiency with lower OLR, and lowest COD removal efficiency with highest OLR (Figure 5). S. ureilytica with OLR1 showed highest COD removal efficiency (84\%), followed by OLR2 (72\%), OLR3 (67\%) and OLR4 (57\%).

Dehydrogenase enzyme activity: Dehydrogenase (DH) enzyme involves in the transfer of $\mathrm{H}^{+}$between metabolic intermediates 
Citation: Venkateswar Reddy M, Venkata Mohan S (2015) Polyhydroxy alkanoates Production by Newly Isolated Bacteria Serratia ureilytica Using Volatile Fatty Acids as Substrate: Bio-Electro Kinetic Analysis. J Microb Biochem Technol 7: 026-032. doi:10.4172/1948-5948.1000177

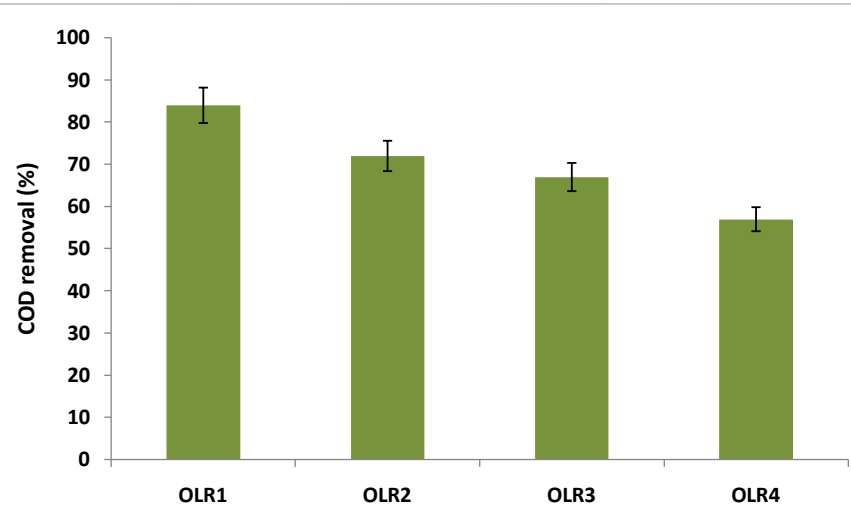

Figure 5: Chemical oxygen demand removal efficiency of Serratiaureilytica at the end of reaction time $(48 \mathrm{~h})$ with different organic loading rate conditions.

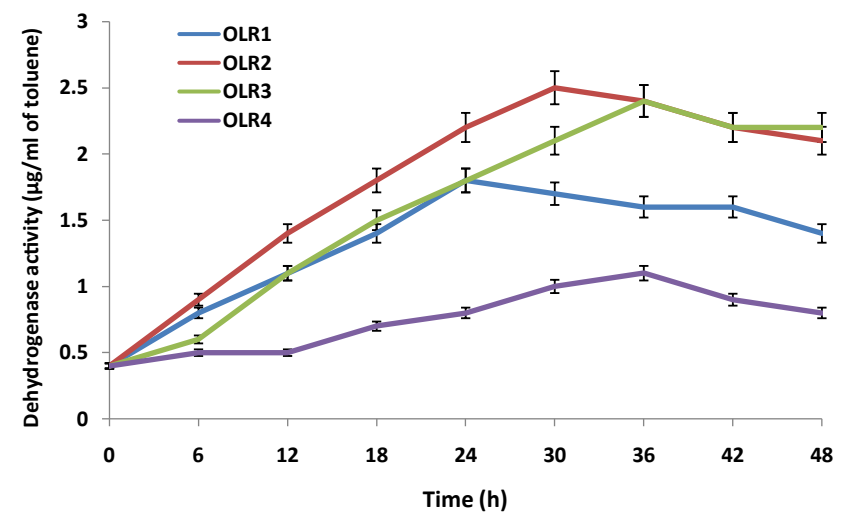

Figure 6: Variation in dehydrogenase enzyme activity of Serratiaureilytica with respect to different time intervals at different organic loading rates.

through redox reactions using mediators like $\mathrm{NAD}^{+}$and $\mathrm{FAD}^{+}$. It is an intracellular enzyme essential for the oxidation and reduction of organic matter. It also involves in the conversion of acetate to acetyl$\mathrm{CoA}$, which is further converted to PHA. Initial DH activity $(0 \mathrm{~h}, 0.6 \mu \mathrm{g} /$ $\mathrm{ml}$ ) was same for all the OLRs studied. DH activity increased with time and then showed decrement which might be due to lower availability of substrate (Figure 6). Among all the OLRs, OLR2 showed maximum enzyme activity $(24 \mathrm{~h}, 2.9 \mu \mathrm{g} / \mathrm{ml})$ followed by OLR3 $(36 \mathrm{~h}, 2.3 \mu \mathrm{g} / \mathrm{ml})$,

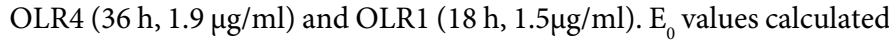
from the redox pair in cyclic voltammetry analysis correspond to $\mathrm{FADH}_{2}$. The presence of $\mathrm{FADH}_{2}$ in cyclic voltammetry analysis at $24 \mathrm{~h}$ and $36 \mathrm{~h}$ confirmed that the dehydrogenase enzyme (acetoacetyl-CoA reductase) is present in the system and involved in the PHA production process. Higher $\mathrm{DH}$ activity observed at $24 \mathrm{~h}$, corroborated well with the high PHA production, good substrate degradation and high reduction current in $\mathrm{CV}$. Higher DH activity increases the intracellular ratio of $\mathrm{NADH} / \mathrm{NAD}^{+}, \mathrm{FADH} / \mathrm{FAD}^{+}$which controls the flux of acetyl-CoA to PHA biosynthetic pathway and slightly reduces the TCA cycle activity. Anderson \& Dawes, (1990) reported that excess NADH/FADH acts as an electron donor in the acetoactyl-CoA reductase catalyzed reaction transforming aceto acetyl-CoA to $\beta$-hydroxybutyryl CoA which could be further converted into PHA by the action of PHA synthase $[34,35]$.

\section{Bio-electro kinetic analysis}

Electrochemical analysis was employed to study the bioelectrochemical behavior of $S$. ureilyticaduring PHA production at
OLR2 during different time intervals ( 0 h, $12 \mathrm{~h}, 24 \mathrm{~h}, 36 \mathrm{~h}$ and $48 \mathrm{~h}$ ). Voltammograms visualized significant variation in the electrochemical behavior of $S$. ureilytica (Figure 7a, Table 1). The catalytic current enumerated the shuttling behavior of $\mathrm{H}^{+}$in the system between metabolic intermediates through redox reactions. High oxidationreduction currents were observed at $24 \mathrm{~h}(0.85 \mathrm{nA},-0.87 \mathrm{nA})$, followed by $36 \mathrm{~h}(0.62 \mathrm{nA},-0.67 \mathrm{nA}), 12 \mathrm{~h}(0.38 \mathrm{nA},-0.51 \mathrm{nA}), 48 \mathrm{~h}(0.34 \mathrm{nA}$, $-0.39 \mathrm{nA})$, and $0 \mathrm{~h}(0.24 \mathrm{nA},-0.33 \mathrm{nA})$. At $24 \mathrm{~h}, 36 \mathrm{~h}$ and $48 \mathrm{~h}$ both oxidation and reduction currents were almost same, but $12 \mathrm{~h}$ showed significant variation in both oxidation and reduction currents. $S$. ureilytica showed higher reduction current than oxidation current at $12 \mathrm{~h}$. By observing the electrochemical analysis, it can be concluded that both oxidation and reduction processes are equally occurring during the PHA production process due to sufficient amount of oxygen availability to carry out the re-oxidation of redox mediators. Specific redox signals were observed on the voltammograms taken at $24 \mathrm{~h}$ and $36 \mathrm{~h}$ of operation, while redox peaks were not found in the voltammograms taken at $12 \mathrm{~h}, 48 \mathrm{~h}$, and $0 \mathrm{~h}$ (Figure $7 \mathrm{a}$ ).

Tafel analysis: Evaluation of electrochemical and kinetic parameters pertaining to the biocatalyst behavior with the function of operating conditions will depict understanding of the electron discharge efficiency of the system. Tafel analysis is the electroanalytical technique used for evaluating the biocatalyst behavior based on proton and electron transfer [36]. Simple charge transfer reactions can be exploited by the linear portion of Tafel slope for deriving the number of protons and electrons transferred in the process along with the exchange current density. The semi-empirical Tafel equation can be

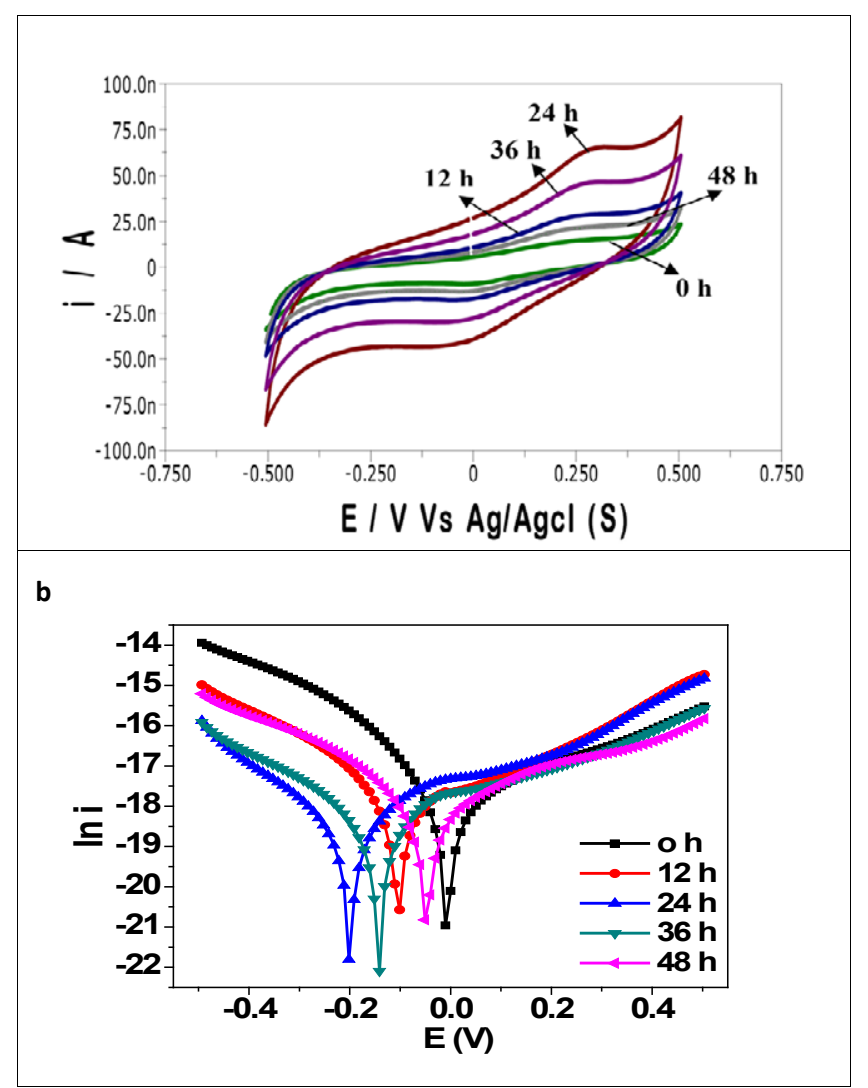

Figure 7: (a) Cyclic voltammograms derived with the function of different time intervals using Serratiaureilytica as biocatalyst; (b) Tafel plots derived from cyclic voltammograms at different time intervals using GPES (4.0) software. 
Citation: Venkateswar Reddy M, Venkata Mohan S (2015) Polyhydroxy alkanoates Production by Newly Isolated Bacteria Serratia ureilytica Using Volatile Fatty Acids as Substrate: Bio-Electro Kinetic Analysis. J Microb Biochem Technol 7: 026-032. doi:10.4172/1948-5948.1000177

\begin{tabular}{|c|c|c|c|c|c|}
\hline \multirow[t]{2}{*}{ Time (h) } & \multicolumn{5}{|c|}{ Tafel Slope (V/dec) } \\
\hline & Oxidation current (nA) & Reduction current (nA) & Oxidative ( $\beta \mathrm{a})$ & Reductive $(\beta c)$ & Polarization resistance $(R p, \Omega)$ \\
\hline 0 & 0.24 & -0.33 & 0.425 & 0.282 & 6.561 \\
\hline 12 & 0.38 & -0.51 & 0.378 & 0.235 & 6.051 \\
\hline 24 & 0.85 & -0.87 & 0.354 & 0.22 & 3.451 \\
\hline 36 & 0.62 & -0.67 & 0.355 & 0.23 & 4.168 \\
\hline 48 & 0.34 & -0.39 & 0.424 & 0.251 & 6.452 \\
\hline
\end{tabular}

Table 2: Bioelectrokinetics analysis of $S$. ureilytica during PHA production with volatile fatty acids at OLR2 operation.

expressed as Equation (1).

$$
\begin{aligned}
& \operatorname{lni}=\mathrm{i}_{0}+\alpha_{\mathrm{a}} \mathrm{nFE} / \mathrm{RT} \\
& \ln \mathrm{i}=\mathrm{i}_{0}-\alpha_{\mathrm{a}} \mathrm{nFE} / \mathrm{RT}
\end{aligned}
$$

Where, i represents current $(\mathrm{A}), \mathrm{E}$ is applied voltage $(\mathrm{V}), \beta \mathrm{a}\left(\alpha_{\mathrm{a}} \mathrm{F} /\right.$ $\mathrm{RT})$ and $\beta c\left(\alpha_{c} \mathrm{nF} / \mathrm{RT}\right)$ are the Tafel slopes. $\alpha_{\mathrm{a}}, \alpha_{c}$ are the electron transfer coefficients, $\mathrm{n}$ is the number of electrons transfer, $\mathrm{R}$ is the universal gas law constant $\left(8.314 \mathrm{~J} \mathrm{~mol}^{-1}-\mathrm{K}\right)$ and $\mathrm{F}$ is the Faraday constant $(96483 \mathrm{C}$ $\left.\mathrm{mol}^{-1}\right)$. A Tafel plot was constructed with each time interval to calculate the kinetic parameters such as oxidative slope $(\beta a)$, reductive slope $(\beta c)$ and polarization resistance $(\mathrm{Rp}$ in $\Omega$ ) (Figure $7 \mathrm{~b}$, Table 2). Lower Tafel slope indicates higher electrocatalytic activity along with electron transfer efficiencies and vice versa. Oxidative slopes were dominant than reductive slopes irrespective of the time indicating the feasibility for higher reduction reactions. However, the slope varied based on the time interval. Higher reductive slope was observed at $0 \mathrm{~h}(0.282 \mathrm{~V} /$ $\mathrm{dec})$, followed by $48 \mathrm{~h}(0.251 \mathrm{~V} / \mathrm{dec}), 12 \mathrm{~h}(0.235 \mathrm{~V} / \mathrm{dec}), 36 \mathrm{~h}(0.230$ $\mathrm{V} / \mathrm{dec})$ and $24 \mathrm{~h}(0.220 \mathrm{~V} / \mathrm{dec})$, indicating higher reduction feasibility at $24 \mathrm{~h}$ (Figure $7 \mathrm{~b}$ ). Oxidative slope also followed the same trend, $0 \mathrm{~h}$ showed higher slope $(0.425 \mathrm{~V} / \mathrm{dec})$, followed by $48 \mathrm{~h}(0.424 \mathrm{~V} / \mathrm{dec})$, $12 \mathrm{~h}(0.378 \mathrm{~V} / \mathrm{dec}), 36 \mathrm{~h}(0.355 \mathrm{~V} / \mathrm{dec})$ and $24 \mathrm{~h}(0.354 \mathrm{~V} / \mathrm{dec})$ (Figure $7 \mathrm{~b})$. Among all the time intervals, $24 \mathrm{~h}$ of operation depicted significant lower redox Tafel slopes compared to other time intervals indicating the higher favorability for both the oxidation and reduction reactions. Generation of protons and electrons from the substrate metabolism followed by their reduction in the PHA production pathway might have contributed for the higher redox reactions depicting lower redox Tafel slopes. Polarization resistance calculated from the Tafel analysis was relatively low at $24 \mathrm{~h}(3.451 \Omega)$, followed by $36 \mathrm{~h}(4.168 \Omega), 12 \mathrm{~h}$ $(6.051 \Omega), 48 \mathrm{~h}(6.452 \Omega)$ and $0 \mathrm{~h}(6.561 \Omega)$ (Table 2). Low polarization resistance observed at $24 \mathrm{~h}$ supports the transfer of higher number of reducing equivalents from biocatalyst towards reduction which might be the PHA production pathway. In cyclic voltammetry analysis $\mathrm{E}_{0}$ values calculated from the redox pair correspond to $\mathrm{FAD}^{+} / \mathrm{FADH}_{2}$ $\left(\mathrm{E}_{0}=-0.24 \mathrm{~V}\right)$. Redox pairs observed during various time intervals suggested the reversible nature of redox reactions. Among all the time intervals $S$. ureilyticaat 24 h showed higher PHA production, high $\mathrm{DH}$ activity and substrate degradation which strongly support the observed polarization resistance. Tafel analysis helped in understanding the variations in the number of electrons present as well as electron transfer resistance during PHA production.

\section{Conclusions}

Bioplastics production with a newly isolated bacterial strain $S$. ureilytica by utilizing volatile fatty acids was successfully evaluated in this study. Bioplastics produced from S. ureilytica contains the copolymer $\mathrm{P}$ (3HB-co-3HV) with high hydroxybutyrate content. Substrate concentration significantly influenced bioplastics accumulation capacity of $S$. ureilytica. Enzyme activities, bioprocess monitoring and substrate degradation showed good correlation with PHA production.
Bio-electro kinetic analysis showed decrement in internal losses during PHA production favored for more electron discharge which might involved in the reduction process. Present study depicted optimum concentration of substrate for higher PHA production in S. ureilytica.

\section{References}

1. Sukan A, Roy I, Keshavarz T (2014) Agro-industrial waste materials as substrates for the production of Poly(3-hydroxybutyric acid). J Biomat Nanobiotechnol 5: 229-240.

2. EPA, (1998) Environmental fact sheet municipal solid waste generation, recycling, and disposal in the United States: facts, and figures for 1998 EPA530-F-00-024, 2000.

3. Madsen EL, (2008) Environmental Microbiology: From Genomes to Biogeochemistry. Wiley Blackwell. Malden MA, 479.

4. Salehizadeh H1, Van Loosdrecht MC (2004) Production of polyhydroxyalkanoates by mixed culture: recent trends and biotechnological importance. Biotechnol Adv 22: 261-279.

5. Hazer B1, Steinbüchel A (2007) Increased diversification of polyhydroxyalkanoates by modification reactions for industrial and medical applications. Appl Microbiol Biotechnol 74: 1-12.

6. Akaraonye E, Keshavarz T, Roy I (2010) Production of polyhydroxyalkanoates: the future green materials of choice. J Chem Technol Biotechnol 85: 732-743.

7. Philip S, Keshavarz T, Roy I (2007) Polyhydroxyalkanoates: Biodegradable polymers with a range of applications. J Chem Technol Biotechnol 82: 233-247.

8. Keshavarz T, Roy I (2010) Polyhydroxyalkanoates: bioplastics with a green agenda. Curr Opin Microbiol 13: 321-326.

9. Valappil SP, Rai R, Bucke C, Roy I (2008) Polyhydroxyalkanoate biosynthesis in Bacillus cereus SPV under varied limiting conditions and an insight into the biosynthetic genes involved. J Appli Microbiol 104: 1624-1635.

10. Venkateswar Reddy M, Amulya K, Rohit MV, Sarma PN, Venkata Mohan S (2014) Valorization of fatty acid waste for bioplastics production using Bacillus tequilensis: Integration with dark-fermentative hydrogen production process. Int J Hydrogen Eneergy 39: 7616-7626.

11. Amulya K, Venkateswar Reddy M, Venkata Mohan S (2014) Acidogenic spent wash valorization through polyhydroxyalkanoate (PHA) synthesis coupled with fermentative biohydrogen production. Bioresour Technol1 58: 336-342.

12. Ryu HW, Hahn SK, Chang YK, Chang HN (1997) Production of poly(3 hydroxybutyrate) by high cell density fed-batch culture of Alcaligenes eutrophus with phospate limitation. Biotechnol Bioeng 55: 28-32.

13. Mozumder MS, Goormachtigh L, Garcia-Gonzalez L, De Wever H, Volcke El (2014) Modeling pure culture heterotrophic production of polyhydroxybutyrate (PHB). Bioresour Technol 155: 272-280.

14. Garcia-Gonzalez L, Mozumder SI, Dubreuil M, Volcke EIP, DeWever (2014) Sustainable autotrophic production of polyhydroxybutyrate (PHB) from CO2 using a two-stage cultivation system. Cat Today: doi.org/10.1016/j. cattod.2014.05.025, (2014)

15. Kim BS, Lee SY, Chang HN (1992) Production of poly-betahydroxybutyrate by fed-batch culture of recombinant Escherichia coli. Biotechnol Lett 14: 811-816.

16. Wang J, Yu J (2000) Kinetic analysis on inhibited growth and poly(3hydroxybutyrate) formation of Alcaligenes eutrophus on acetate under nutrientrich conditions. Proc Biochem 36: 201-208.

17. Sharma L, Mallick N (2005) Enhancement of poly-b-hydroxybutyrate 
Citation: Venkateswar Reddy M, Venkata Mohan S (2015) Polyhydroxy alkanoates Production by Newly Isolated Bacteria Serratia ureilytica Using Volatile Fatty Acids as Substrate: Bio-Electro Kinetic Analysis. J Microb Biochem Technol 7: 026-032. doi:10.4172/1948-5948.1000177

accumulation in Nostoc muscorum under mixotrophy, chemoheterotrophy and limitations of gas-exchange. Biotechnol Lett 27: 59-62.

18. Albuquerque MGE, Martino V, Pollet E, Averous L, Reis MAM (2011) Mixed culture polyhydroxyalkanoate (PHA) production from volatile fatty acid (VFA)rich streams: Effect of substrate composition and feeding regime on PHA productivity, composition and properties. J Biotechnol 151: 66-76.

19. Venkata Mohan S, Venkateswar Reddy M (2013) Optimization of critical factors to enhance polyhydroxyalkanoates (PHA) synthesis by mixed culture using Taguchi design of experimental methodology. Bioresour Technol 128: 409-416.

20. Venkateswar Reddy M, Nikhil GN, Venkata Mohan S, Swamy YV, Sarma PN (2012) Pseudomonas otitidis as a potential biocatalyst for polyhydroxyalkanoates (PHA) synthesis using synthetic wastewater and acidogenic effluents. Bioresour Technol 123: 471-479.

21. APHA (1998) Standard methods for the examination of water and wastewater. (20th edition). American Public Health Association/American water works Association/Water environment federation, Washington DC, USA.

22. Lv Z, Yao Y, Lv Z, Min H (2008) Effect of tetrahydrofuran on enzyme activities in activated sludge. Ecotoxicol Environ Saf 70: 259-265.

23. LAW JH, SLEPECKY RA (1961) Assay of poly-beta-hydroxybutyric acid. J Bacteriol 82: 33-36.

24. Venkateswar Reddy M, Venkata Mohan S (2012) Influence of aerobic and anoxic microenvironments on polyhydroxyalkanoates (PHA) production from food waste and acidogenic effluents using aerobic consortia. Bioresour Technol 103: 313-321.

25. Karr DB, Waters JK, Emerich DW (1983) Analysis of Poly-beta-Hydroxybutyrate in Rhizobium japonicum Bacteroids by Ion-Exclusion High-Pressure Liquid Chromatography and UV Detection. Appl Environ Microbiol 46: 1339-1344.

26. Jayarama Reddy S (1986) Studies on electrode processes by cyclic voltammetry, chronoamperometry, chronopotentiometry and controlled potentaial coulometry. Sri Venkateswara University, Tirupati.

27. Du G, Si Y, Yu J (2011) Inhibitory effect of medium-chain-length fatty acids on synthesis of polyhydroxyalkanoates from volatile fatty acids by Ralstonia eutropha. Biotechnol Lett 23: 1613-1617.

28. Lugg H, Sammons RL, Marquis PM, Hewitt CJ, Yong P, et al. (2008) Polyhydroxybutyrate accumulation by a Serratia sp. Biotechnol Lett 30: 481491.

29. Nalini S, Parthasarathi $R$ (2014) Production and characterization of rhamnolipids produced by Serratia rubidaea SNAU02 under solid-state fermentation and its application as biocontrol agent. Bioresour Technol 173: 231-238.

30. Wang SL, Lin CL, Liang TW, Liu KC, Kuo YH (2009) Conversion of squid pen by Serratia ureilytica for the production of enzymes and antioxidants. Bioresour Technol 100: 316-323.

31. Bhadra B, Roy P, Chakraborty R (2005) Serratia ureilytica sp. nov., a novel urea-utilizing species. Int J Syst Evol Microbiol 55: 2155-2158.

32. Yee LN, Mumtaz T, Mohammadi M, Phang LY, Ando Y, et al. (2012) Polyhydroxyalkanoate synthesis by recombinant Escherichia coli JM109 expressing PHA biosynthesis genes from Comamonas sp. EB172. J Microbial Biochem Technol 4: 103-110.

33. Lee SY (1996) Bacterial polyhydroxyalkanoates. Biotechnol Bioeng 49: 1-14.

34. Anderson AJ1, Dawes EA (1990) Occurrence, metabolism, metabolic role, and industrial uses of bacterial polyhydroxyalkanoates. Microbiol Rev 54: 450-472.

35. Brigham CJ, Kurosawa K, Rha CK, Sinskey AJ (2011) Bacterial carbon storage to value added products. J Microbial Biochem Technol: S3:002.

36. Venkata Mohan S, Srikanth S (2011) Enhanced wastewater treatmen efficiency through microbially catalyzed oxidation and reduction: synergistic effect of biocathode microenvironment. Bioresour Technol 102: 10210-10220. 